\title{
Estrategia de Intervención digital basada en el constructivismo para el conocimiento de lavado de manos en estudiantes de enfermería
}

\section{Digital intervention strategy based on constructivism for the knowledge of hand washing in nursing students}

\section{Estratégia de intervenção digital baseada no construtivismo para o conhecimento da desinfecção das mãos em estudantes de enfermagem}

\author{
Marco Esteban Morales-Rojas ${ }^{1}$ \\ Sheila Mariela Cohuo-Cob ${ }^{2}$ \\ Didier Francisco Aké-Canul ${ }^{3}$ \\ Russel Izael Trujeque-Zavala ${ }^{4}$ \\ Marianely Pech-Irola ${ }^{5}$
}

\begin{abstract}
Resumen
Introducción: La higiene de manos es toda técnica destinada a remover, destruir o reducir la flora transitoria de la piel. Diversas organizaciones concuerdan en que es el procedimiento más costo-efectivo que puede disminuir las infecciones en pacientes hospitalizados, así como proteger al personal que se encuentra en contacto con ellos. Actualmente, ante los retos provocados por la pandemia de la COVID-19, las técnicas de enseñanza-aprendizaje optan por modalidades tecnológicas a distancia con enfoques basados en la autogestión del conocimiento y el desarrollo de habilidades propias, que puedan incidir en este hábito. $\boldsymbol{O} \boldsymbol{b}$ jetivo: Evaluar la efectividad de una intervención digital con enfoque constructivista para aumentar el conocimiento del lavado de manos. Metodología: Estudio cuasi experimental, longitudinal y prospectivo. Se seleccionaron a 26 estudiantes de enfermería del último año de un programa educativo a través de un muestreo no probabilístico a conveniencia, con quienes se llevó a cabo una intervención virtual con enfoque constructivista. A tal efecto se utilizó el instrumento "Conocimiento sobre higiene de manos en profesionales sanitarios" (alfa de Cronbach $=0.784$ ) para la prueba de diferencia de conocimiento pre y post intervención. $\boldsymbol{R} \boldsymbol{e}$ sultados: Posterior a la intervención, el 100\% de los sujetos reconocen las manos como principal vía de transmisión y el $73.10 \%$ identifica correctamente el tiempo necesario para llevarlo a cabo; sin embargo, no existe diferencia estadísticamente significativa del conocimiento previo. Conclusión: A través de las intervenciones constructivistas los estudiantes tienen la oportunidad de ser gestores de su propio conocimiento y adquirir conductas, hábitos y experiencias superiores a los objetivos planteados; no obstante, se debe tomar en cuenta los factores de contextualización y operatividad de las intervenciones para lograr su mayor efectividad.
\end{abstract}

Palabras clave: Lavado de manos; intervención digital; estudiantes de enfermería.
Autor de correspondencia*

${ }^{1 *}$ Lic. En Enfermería. Maestro en Salud Pública. Profesor de Educación Superior. Universidad Autónoma de Yucatán. Mérida, Yucatán, México. Correo: mar-

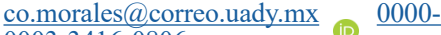
0003-3416-0806

2 Lic. En Enfermería. Maestra en Gobierno y Políticas Públicas. Profesora de Educación Superior. Universidad Autónoma de Yucatán. Mérida, Yucatán, México. Correo: sheila.cohuo@ correo.uady.mx (iD) 0000-0003-4936$\underline{5142}$

${ }^{3}$ Lic. En Enfermería. Maestro en Salud Pública. Profesor de Educación Superior. Universidad Autónoma de Yucatán. Secretario Administrativo de la Facultad de Enfermería. Mérida, Yucatán, México. Correo: didier.ake@correo. uady.mx (D) 0000-0001-8089-9156

${ }^{4}$ Lic. En Trabjo Social. Profesor de Educación Superior. Universidad Autónoma de Yucatán. Mérida, Yucatán, México. Correo: russell.trujeque@,correo. uady.mx (1) 0000-0001-7575-1245

${ }^{5}$ Lic. En Enfermería. Profesora de Educación Superior. Universidad Autónoma de Yucatán. Mérida, Yucatán, México. Correo: marianely.pech@,correo.uady. $\underline{\mathrm{mx}}$ (1) 0000-0001-6852-6744

Recibido: 14 abril 2021

Aprobado: 10 agosto 2021

Introduction: Hand hygiene is any technique designed to remove, destroy, or reduce the transient flora of the skin. Many organizations agree that this is the most cost-effective procedure that can prevent hospital-acquired infections in hospitalized patients, as well as protecting the personnel that is in contact with them. Currently, facing the challenges caused by the COVID-19 pandemic, the teaching-learning techniques opt for distance technological modalities with approaches based on self-management of knowledge and the development of 
self- skills, which can have an impact on this habit. Objective: Evaluate the effectivity of a digital intervention with a constructivist approach to increase the knowledge of handwashing. Methodology: Quasi-experimental, longitudinal, and prospective study. Twenty-six nursing students in the last year of an educational program were selected through a non-probability convenience sample, which had a virtual intervention with a constructivist approach. To that effect the instrument used was "Knowledge about hand hygiene in healthcare professionals" (Cronbach's alpha $=0.784$ ) to test the difference of knowledge pre and post intervention. Results: After the intervention, $100 \%$ of the subjects recognize that hands are the main way of transmission and $73.10 \%$ correctly identify the time needed to wash their hands; however, there is no significant statistical difference of previous knowledge. Conclusion: Through constructivist interventions the students have the chance of managing their own knowledge and acquire behaviors, habits and experiences beyond the objectives set; nevertheless, factors of contextualization and operativity should be considered for the interventions to achieve greater effectivity.

Keywords: Handwashing; digital intervention; nursing students.

\section{Para citar este artículo}

Morales-Rojas ME, Cohuo-Cob SM, Aké-Canul DF, Trujeque-Zavala RI, Pech-Irola M. Estrategia de Intervención digital basada en el constructivismo para el conocimiento de lavado de manos en estudiantes de enfermería. Rev. cienc. cuidad. 2021; 18(3):54-63. https:// doi.org/10.22463/1794983i.2916

(C) Universidad Francisco de Paula Santander. Este es un artículo bajo la licencia CC-BY-NC-ND

\section{Resumo}

Introdução: A higiene das mãos é toda técnica destinada à remoção, destruição ou redução da flora transitória da pele. Diversas organizações concordam que é o procedimento mais custo-benefício que pode reduzir as infeções no paciente internado, assim como proteger o pessoal que está em contato com eles. Atualmente, os desafios provocados pela pandemia da COVID-19, as técnicas de ensino têm acolhido ferramentas tecnológicas a distância com fundamentação na autogestão do conhecimento e o desenvolvimento de habilidades próprias, que possam incidir nesse habito. Objetivo: Avaliar a efetividade de uma intervenção digital com fundamentação construtivista para incrementar o conhecimento do lavado das mãos. Métodos: Estudo quase-experimental, longitudinal, prospetivo. Foram escolhidos 26 alunos de enfermagem de último ano através de uma amostragem não probabilística por conveniência. Foi feita uma intervenção virtual empregando-se o instrumento "Conhecimento sobre higiene das mãos em profissionais sanitários" (alfa de Cronbach $=0,78$ ) para a prova de conhecimento pre e pós intervenção. Resultados: Posterior à intervenção, 100\% dos participantes reconhecem as mãos como a principal fonte de transmissão e $73,10 \%$ identifica corretamente o tempo necessário para lavar as mãos; entretanto, não existe diferença significativa do conhecimento prévio. Conclusão: Através das intervenções construtivistas os alunos têm a oportunidade de ser gestores do seu próprio conhecimento e adquirir condutas, hábitos e experiências superiores aos objetivos formulados; entretanto, deve-se considerar os fatores como contexto e operabilidade das intervenções para conseguir a maior efetividade.

Palavras-chave: Desinfecção das mãos; educação à distância; estudantes de enfermagem.

\section{Introducción}

El lavado de manos (LM) es el término asignado a toda técnica o procedimiento destinado a remover la suciedad y microorganismos transitorios de la piel por acción mecánica, a través de la fricción con jabón y agua abundante durante un tiempo mínimo de 20 segundos (1). En el personal sanitario, las manos constituyen un vehículo importante para la transmisión de agentes patógenos entre pacientes y colegas en el contexto hospitalario, propenso al contagio de infecciones asocia- das a la atención sanitaria (IAAS) (2). Actualmente, la Organización Mundial de la Salud estima que el costo relacionado con estas afectaciones asciende aproximadamente a unos 1.500 millones de dólares al año, debido al incremento en gastos farmacéuticos, la estancia en el hospital y las pérdidas relacionadas con la incapacidad laboral; además, se evidencia una calidad ineficiente en la prestación de cuidados sanitarios (3).

En México, las IAAS son de alta comorbilidad y mortalidad en los pacientes hospitalizados por infecciones 
en vías urinarias, con intervenciones quirúrgicas y neumonías asociadas con la ventilación mecánica (4). El personal de la enfermería a través de sus distintas funciones puede ser una fuente de contaminación, por lo que el lavado de manos antes y después de cada procedimiento es un componente clave, simple, económico y aplicable en la práctica diaria para prevenir contagios (5). En el actual contexto de la pandemia por COVID-19 se han implementado medidas preventivas como el distanciamiento social, el uso de mascarillas y reforzamiento del lavado de manos.

Con respecto a ésta última medida, las estrategias de contención se han enfocado principalmente a educar e informar sobre dicha práctica, tanto a personas pertenecientes al sector de la salud como a la población en general; sin embargo, la adherencia y cumplimiento de esta técnica dista de replicarse de forma óptima (6). Como miembros activos del personal de salud, el de la enfermería debe reconocer la necesidad no solo de participar en el establecimiento de mecanismos permanentes del equipo de vigilancia epidemiológica, sino también como líderes y pieza clave en la promoción, conservación y establecimiento de conductas en salud que repercutan directamente en el bienestar del paciente (7).

En este sentido, los estudiantes de enfermería, a través de las prácticas en escenarios reales de aprendizaje se exponen a múltiples gérmenes en el ambiente clínico $\mathrm{y}$ esto los convierte en potenciales portadores y transmisores de dichos patógenos a otros pacientes, compañeros e incluso a sus familiares en los hogares (8). Estudios demuestran que existen lagunas de conocimiento en relación con el LM dentro de la etapa formativa del personal de la enfermería; por tal motivo, es importante que los programas educativos fortalezcan en cada nivel la importancia, calidad, conocimientos y actitudes relacionadas con dicha técnica, no solo como medida preventiva de las Infecciones Asociadas a la Atención en Salud, sino como un indicador importante en la calidad de los cuidados hospitalarios $(9,10)$.

Bajo este concepto se han instaurado programas de educación en el lavado de manos de los trabajadores hospitalarios principalmente enfocados en la calidad, el tiempo y los momentos de implementación; no obstante, son escasas las publicaciones centradas en el método de su aplicación y el enfoque educativo. Al respecto, el constructivismo como corriente pedagógica indica que las estrategias de enseñanza aprendizaje sean dinámicas y posiciona al estudiante como centro de los sistemas, capaz de resolver situaciones problema a través de la construcción de herramientas y conocimientos propios. Derivado de esta corriente, el aprendizaje basado en problemas ofrece técnicas grupales, en donde cada integrante adquiere la responsabilidad colectiva para resolver una problemática. Además del conocimiento, se propicia la oportunidad de trabajar en las competencias relacionadas con la comunicación efectiva, las actitudes y valores asociados y un proceso de realimentación constante $(11,12)$.

En el contexto de la pandemia por COVID-19, las modalidades de enseñanza se han modificado en apego a las medidas de prevención, provocando una transición apresurada hacia los programas educativos de modalidad presencial a la virtual. En la modalidad virtual el estudiante es guiado por el docente, quien facilita herramientas como redes sociales, medios de búsqueda de información de calidad y entornos colaborativos digitales que le permiten al estudiante alcanzar los conocimientos y desarrollar competencias $(13,14)$.

En función de lo expuesto anteriormente, se plantea el siguiente proyecto, cuyo objetivo es el de evaluar la efectividad de una intervención digital con enfoque constructivista para aumentar el conocimiento del lavado de manos.

\section{Material y métodos}

Este es un estudio cuasi experimental de tipo pre-post test, derivado de un macroproyecto titulado Intervención multicomponente para promover el lavado de manos en escuelas primarias públicas de Yucatán. Se seleccionó a un grupo de 26 estudiantes del octavo semestre (último) de la licenciatura en enfermería, a través de un muestreo no probabilístico a conveniencia. Se tuvieron en cuenta como criterios de inclusión a los estudiantes que hubiesen completado los campos prácticos previos, y que estuviesen inscritos de forma regular (que no tengan asignaturas no acreditadas) durante el 2020.

Para la medición se utilizó el instrumento "Conocimiento sobre higiene de manos en profesionales sanitarios" creado por el Ministerio de Sanidad, Política Social e Igualdad de España y utilizado por la Subsecretaría de Integración y Desarrollo del Sector Salud, dependiente de la Secretaría de Salud en México. Tiene como puntuación máxima 21 aciertos (sin contar las preguntas relativas a las características personales y educativas). Dicho instrumento ha sido utilizado por autores como González, Fernández y Trujillo obteniendo una confiabilidad de 0.784 con alfa de Cronbach; evalúa 4 áreas principales: fuentes de contaminación, momentos para el lavado de manos, conocimientos sobre lavado de manos con alcohol gel/agua jabón y cuidados alrededor del lavado de manos $(15,16)$. 


\section{CienciáoCuidado}

Scientific Journal of Nursing
El estudio se llevó a cabo en un periodo de 3 meses, de julio a septiembre de 2020. La primera medición se efectuó a través del instrumento previamente descrito, el cual se realizó el 13 de julio; posteriormente se aplicó la intervención basada en diversas técnicas propuestas por la corriente constructivista, recopiladas a través de manuales propios de la institución y adaptadas a experiencias como la de Garzón, Bautista y Morales (17). Estas propuestas se identificaron con el aprendizaje basado en problemas y en casos reales, que a continuación se describen:

13 de julio. Exposición virtual, a cargo de los investigadores, de 1.5 horas, donde se abarcó la definición de los conceptos y diferenciación entre los tipos de higiene de manos (lavado y desinfección), bases biológicas y fisiológicas, normas internacionales, momentos clave, procedimientos y precauciones relativas al cuidado.

10 de agosto. Aprendizaje colaborativo. Al formar equipos de 5 personas, los estudiantes analizaron un caso clínico, referentes a los momentos críticos del lavado de manos, en función de: higiene vs lavado y los cuidados alrededor del procedimiento.

17 de agosto. Aprendizaje basado en problemas. En equipos, los participantes diseñaron una intervención para resolver el problema planteado en el caso clínico como parte de una valoración familiar (con el formato de Proceso Enfermero), teniendo como objetivo central la enseñanza del procedimiento del lavado de manos.

20 de agosto. Diseño de materiales audiovisuales. Como estrategia de evaluación propia, los estudiantes diseñaron un video, que sería implementado como parte de su intervención del aprendizaje basado en problemas, en el mismo demostraron los aspectos esenciales del lavado de manos y su realización.
Por último, se esperó un periodo de 15 días posteriores al último componente de la intervención educativa y se midió el conocimiento con el mismo instrumento, aleatorizando el orden de los reactivos. En relación con los lineamientos éticos y legales, el presente trabajo cumple con los apartados del Reglamento de la Ley General de Salud en Materia de Investigación para la Salud, que, en el Título Segundo, Artículo 17 lo considera sin riesgo. Es necesario precisar, que los participantes dieron su consentimiento informado y el proyecto fue aprobado por el Comité de ética e Investigación de la dependencia correspondiente.

Para el análisis estadístico se utilizaron los programas de Microsoft Excel para medidas de tendencia central y de dispersión, a fin de describir los variables. Con el SPSS V.21 se realizó la prueba t de student con el objeto de cuantificar la diferencia de conocimientos en el grupo intervenido

\section{Resultados}

Se establece que la edad media de los 26 estudiantes es de 23.04 años; el $84.6 \%$ (22) son mujeres y el $15.4 \%$ (4) son hombres. De igual manera, el 50\% (13) eran de la capital del estado, mientras que el resto provenía de municipios del interior del país, contando con un 42.3\% (11) y un $7.7 \%$ (2) de los estados vecinos. En cuanto el último servicio donde los estudiantes realizaron sus prácticas se reportaron los siguientes escenarios: cuidados intensivos (46.2\%), Medicina interna (7.7\%), pediatría $(7.7 \%)$, obstetricia $(7.7 \%)$, quirófanos $(3.8 \%)$ y otros $(26.9 \%)$.

Las respuestas indican que el 100\% recibió al menos una capacitación sobre este cuidado en los últimos 3 años. A continuación, se presentan los resultados de la evaluación pre y post intervención.

Tabla 1. Conocimientos sobre fómites relacionados a las infecciones asociadas a la atención en salud (IAAS).

\begin{tabular}{llc}
\hline Conocimientos relacionados con fómites & & Preprueba \\
\hline Variable & Posprueba \\
Principal vía de transmisión cruzada de microorganismos & $88.50 \%$ & $100 \%$ \\
- Las manos de los profesionales cuando no están limpias & $11.50 \%$ & 0 \\
- La exposición de los pacientes a superficies colonizadas por gérmenes & & $3.80 \%$ \\
Fuente más frecuente de gérmenes causantes de IAAS & $3.80 \%$ & $19.20 \%$ \\
- El sistema de agua del hospital & $15.40 \%$ & $76.90 \%$ \\
- Microorganismos ya presentes en el paciente & $80.80 \%$ & \\
- El entorno (las superficies del hospital) & & \\
\hline
\end{tabular}

Fuente: elaboración propia. 
Al analizar las respuestas del instrumento, en las variables de vía de transmisión (Ver Tabla 1) se determina que en la preprueba más del $80 \%$ de los estudiantes identifica que las manos del personal sanitario es la principal vía de transmisión; en la posprueba se reg- istra un incremento del $11.5 \%$. Después, la respuesta "microorganismos" aumenta en $3.80 \%$, mientras que "el entorno" registra un decremento del $3.90 \%$ en la posprueba.

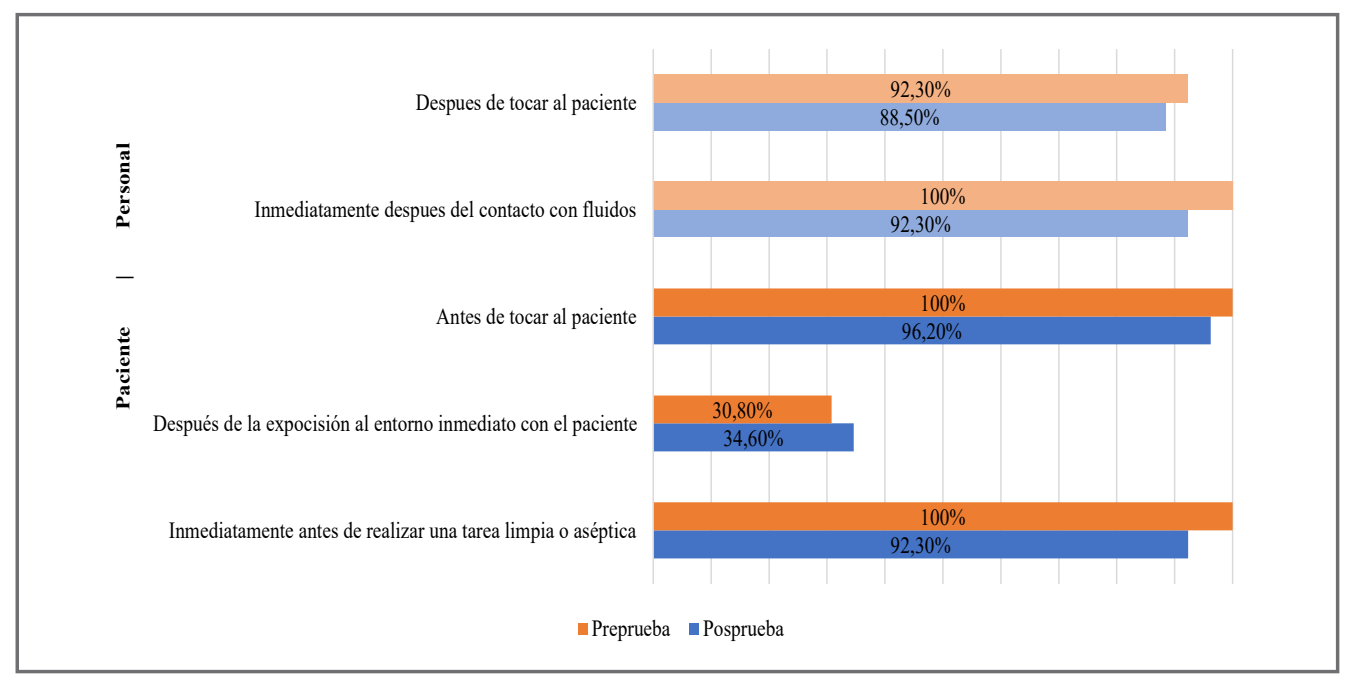

Figura 1. Momentos para realizar la higiene de manos como prevención de la transmisión de microorganismos al paciente-profesional sanitario.

Fuente: elaboración propia.

En segundo lugar, se reporta que el 100\% de los estudiantes identificó en la preprueba que el LM después de estar en contacto con fluidos, antes de tocar al paciente y de realizar una tarea limpia o aséptica, son acciones esenciales para prevenir las infecciones cruzadas. En la posprueba se registró una disminución del 3.80\% para las frases 1 y 3 , para las frases 2 y 5 el decremento fue del $7.70 \%$ y finalmente un incremento del $3.80 \%$ para la frase 4 (Ver Figura 1).

Por otro lado, el $73.1 \%$ de los estudiantes determina como satisfactorio el tiempo necesario para el lavado de manos, mientras que el $23.1 \%$ considera erróneo utilizar 30 segundos y el 3.8\% 10 segundos. No existe variación en los resultados entre la pre y posprueba.

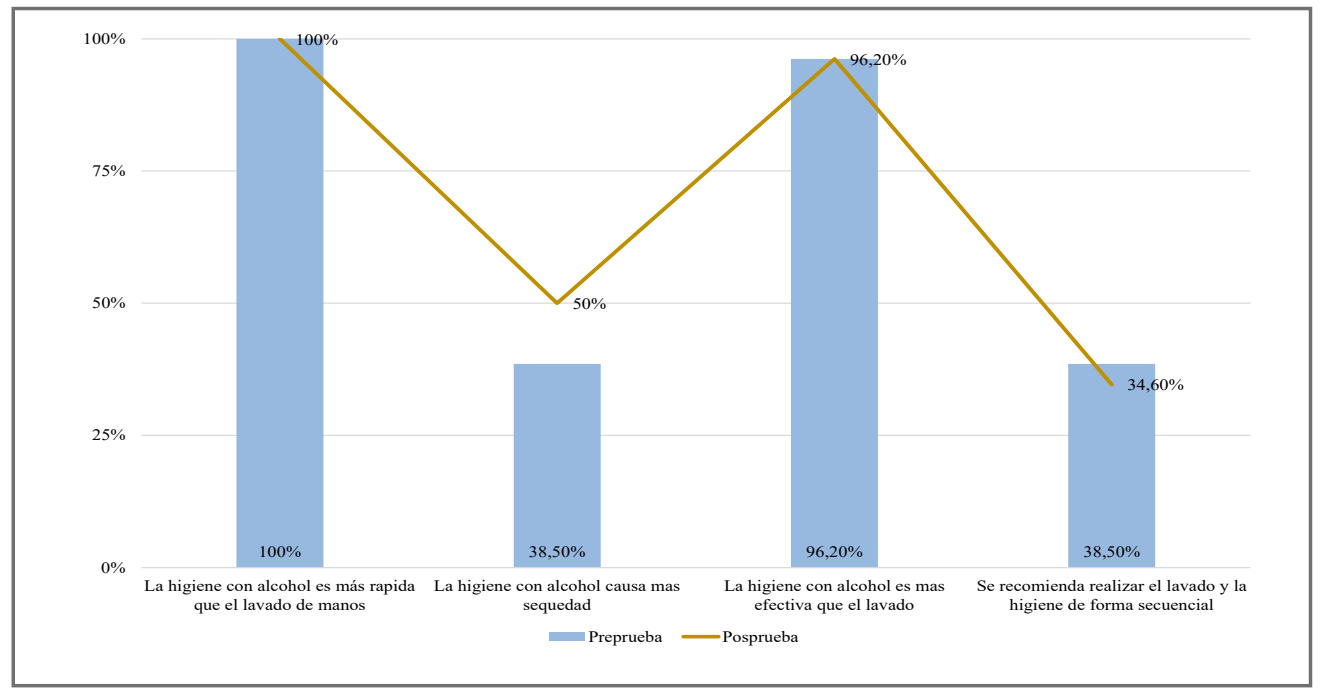

Figura 2. Conocimientos del lavado de manos y la higiene de manos con alcohol gel. Fuente: Elaboración propia. 


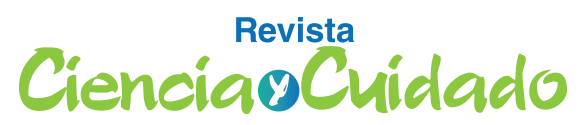

Scientific Journal of Nursing
Al evaluar los conocimientos del lavado e higiene de manos (Ver Figura 2) en la posprueba se registró un incremento del $11.50 \%$ para la segunda afirmación; sin embargo, la mitad de los estudiantes precisó como falso que el alcohol gel causara más sequedad que el lavado con jabón; por otra parte, se observa el decremento del $3.90 \%$ para la cuarta afirmación donde menos del $40 \%$ identifica que no se recomienda la práctica secuencial de lavado y desinfección de manos.

Tabla 2. Elección de la técnica de higiene de manos de acuerdo con los cinco momentos

\begin{tabular}{|c|c|c|c|c|}
\hline \multicolumn{5}{|l|}{ Tipo de higiene de manos según la situación } \\
\hline & \multicolumn{2}{|c|}{ Preprueba } & \multicolumn{2}{|c|}{ Posprueba } \\
\hline & Lavado & Desinfección & Lavado & Desinfección \\
\hline Antes de la palpación del abdomen & $34.60 \%$ & $65.40 \%$ & $34.60 \%$ & $65.40 \%$ \\
\hline Antes de aplicar una inyección & $84.60 \%$ & $15.40 \%$ & $84.60 \%$ & $15.40 \%$ \\
\hline Después de vaciar la orina & $96.20 \%$ & $3.80 \%$ & $96.20 \%$ & $3.80 \%$ \\
\hline Después de retirarse los guantes de examinación & $69.20 \%$ & $30.80 \%$ & $73.10 \%$ & $26.90 \%$ \\
\hline Después de arreglar la cama del paciente & $88.50 \%$ & $11.50 \%$ & $84.60 \%$ & $15.40 \%$ \\
\hline Después de la exposición visible a sangre & $92.30 \%$ & $7.70 \%$ & $100 \%$ & $0.00 \%$ \\
\hline
\end{tabular}

Fuente: Elaboración propia

De acuerdo con el total de sujetos (26) que respondieron la encuesta, se recoge la cantidad de respuestas correctas para cada situación iniciando por la aseveración "antes de la palpación del abdomen", dando como resultados: $17(65 \%), 22(84.60 \%), 25(96.20 \%), 18$
$(69.20 \%), 3(11.50 \%)$ y $24(92.30 \%)$. En la posprueba se registró un incremento del $3.90 \%$ y $7.70 \%$ para las dos últimas aseveraciones respectivamente; para las tres primeras situaciones se mantienen los resultados sin variación entre la pre y posprueba (Ver Tabla 2).

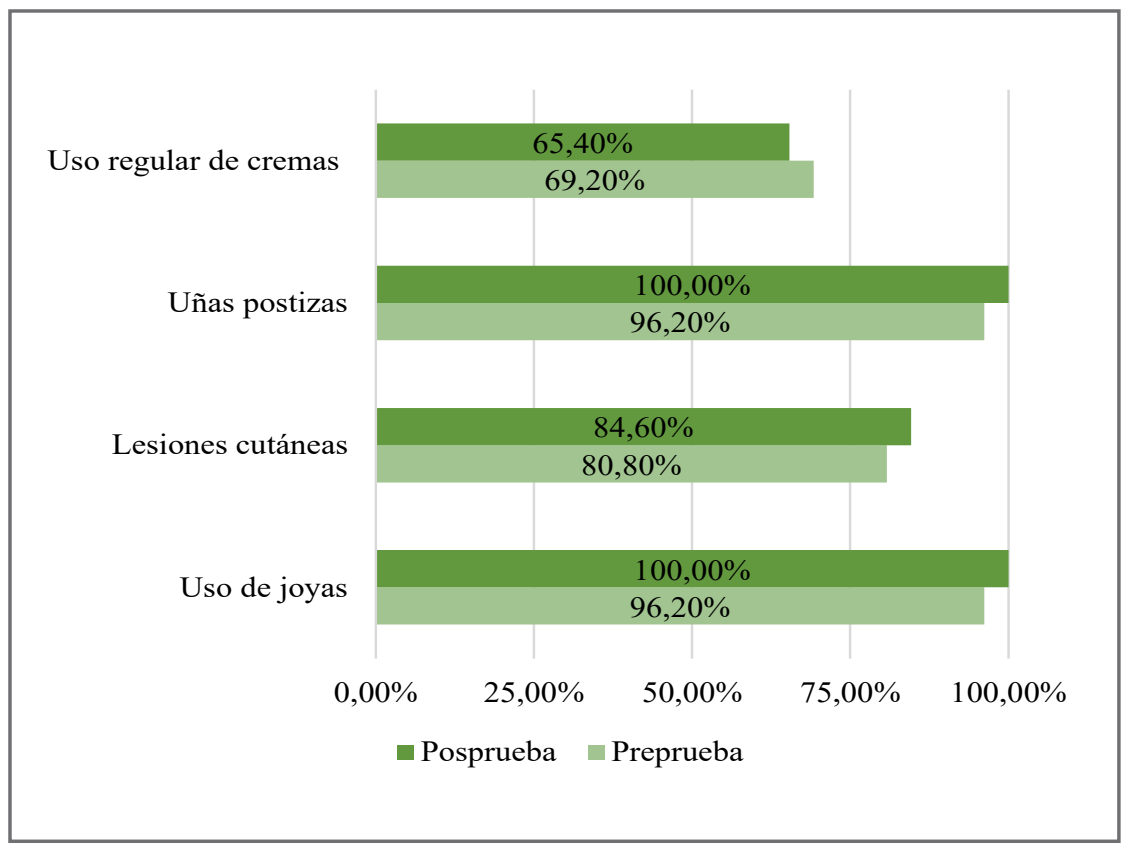

Figura 3. Circunstancias que deben evitarse durante la prestación de servicios de atención a la salud.

Fuente: Elaboración propia. 
Con relación a las circunstancias que deben evitarse se registra un incremento de $3.80 \%$ en los resultados obtenidos en la posprueba para los elementos a evitar: uñas, lesiones y joyas. Menos del 90\% de los estudiantes señala en la pre y posprueba que las lesiones cutáneas durante la atención en salud favorecen la colonización de patógenos (Ver Figura 3).

Finalmente, para la prueba de diferencia de conocimiento se utilizó en primer lugar la prueba de Shapiro-Wilk a fin de corroborar la normalidad de las variables $(\alpha=0.05)$, obteniéndose valores de 0.445 y 0.273 en la preprueba y posprueba, respectivamente. Por lo anterior se utilizó la prueba t de Student como estadístico para evaluar la diferencia de medias. La media de conocimiento general del grupo antes de la intervención fue de 19.73 puntos $(\mathrm{DE}=2.08)$, y posterior a ella se reporta el 19.65 (DE=1.99). Al realizar la prueba t de Student el P valor que se obtuvo es de 0.852 , por lo que se establece de acuerdo con el nivel de confianza $(95 \%)$ que la diferencia no es significativa.

\section{Discusión}

Ante la importancia de fortalecer la capacitación del lavado de manos en estudiantes, debido a los deficientes niveles en el dominio teórico y técnico de la práctica (11), el objetivo de esta investigación consistió en evaluar la efectividad de una intervención educativa basada en el modelo constructivista para mejorar conocimientos, actitudes y cuidado de la higiene de manos en estudiantes de enfermería. Los resultados determinan que el conocimiento se ubicó en un nivel alto considerando un aumento en la posprueba, lo cual difiere con lo reportado por Mamani, quien establece en su estudio que el conocimiento relativo a la transmisión de microorganismos en un nivel regular (44\%) y bueno (42\%), se da especialmente al momento de identificar las manos de los profesionales sanitarios cuando no están limpias (18).

Al evaluar las fuentes causantes de infecciones, Moran informa que en su grupo de intervención el 41.30\% identificó correctamente en el pretest el entorno del paciente como medio principal, mientras que los resultados posteriores a la intervención aumentaron al 62.9\%. Si bien el incremento entre la aplicación previa y posterior también coincide, en los presentes resultados se reporta un aumento del $80.8 \%$ (19). Con relación a la duración de lavado de manos, esta investigación muestra que el nivel de conocimiento se mantuvo constante posterior a la intervención $(73.1 \%)$, en contraste con lo reportado por Korhonen, Vuori y Lukkari, quienes dedujeron en un estudio experimental, que los niveles de conocimiento en este rubro fueron de un $89 \%$ en el grupo intervenido, mientras que en el de control fue de $19 \%$, siendo una de sus variables estadísticamente significativas (20).

Al evaluar la diferencia entre el uso de agua y jabón o la higiene con solución alcohol gel, se observa que los valores de respuestas correctas en esta investigación oscilan entre el $11.50 \%$ y el $96.2 \%$, similar a los hallazgos de Rosales: $62.9 \%$ - 91.4\%. Se debe precisar que las principales diferencias se dan después de retirarse los guantes en un $69.20 \%$ versus $90 \%$ y antes de aplicar una inyección con $84.60 \%$ versus $72.9 \%$, respectivamente (21). En función de las consideraciones previas referidas al lavado de manos con agua y jabón después de la intervención, los estudiantes precisan en su totalidad que se debe evitar el uso de uñas postizas y de joyas, lo cual coincide con lo reportado por González, Fernández y Trujillo, quienes en un estudio transversal evaluaron a 113 profesionales de la salud en un hospital y determinaron que el personal recomienda siempre la precaución de remover el uso de joyas $(22,23)$.

La comparación de los resultados expuestos previamente sobre los conocimientos y recomendaciones relacionados a la práctica del lavado de manos, incluida la temporalidad, medios de trasmisión de microorganismos, principal fuente infecciosa y el discernimiento entre el uso de agua y jabón versus alcohol gel, evidencia la variación de resultados entre el pre-test y pos-test para cada elemento. Sin embargo, al evaluar si la intervención educativa con enfoque constructivista determina un aumento en el conocimiento, la prueba estadística para la diferenciación de medias en los momentos del experimento no reporta un cambio significativo atribuible al componente educacional.

Como se ha dicho para el abordaje de la intervención educativa desde el constructivismo se tomó como punto de partida la capacidad de los estudiantes en la gestión su propio aprendizaje y la adopción de metodologías flexibles, donde el facilitador concede el protagonismo y se responsabiliza de la mediación de los recursos-herramientas y del acompañamiento al estudiante como eje central de la práctica pedagógica (24). En este sentido, al incorporar estrategias como el análisis de casos clínicos, diseño de intervenciones y material educativo, se estructuró un enfoque combinado de aprendizaje-instrucción flexible y auto-gestionable, mediado a través de la educación remota, mediante la transición a través de la modalidad virtual debido a las medidas de distanciamiento social por el COVID-19 (25).

No obstante, el limitado alcance de la intervención para generar cambios significativos en el conocimiento de lavado de manos cuestiona los resultados de Bloom- 
field et al. (26) quienes, en una investigación de control aleatorizado en estudiantes de enfermería, concluyen que el aprendizaje audiovisual asistido por computadora resultó igual de efectivo para enseñar la teoría y práctica del lavado de manos que los métodos convencionales de enseñanza presencial (27).

De su parte, Konicki y Miller discuten esta situación tras reportar que no hubo diferencia significativa entre las medias de los grupos de control e intervención para la sub-escala de conocimiento de lavado de manos, en un estudio basado en la repetición como método de aprendizaje de acuerdo con la teoría social cognitiva y apoyado por sesiones informativas, la visualización de un video referente al tema y la simulación de casos prácticos bajo las recomendaciones de la OMS. En concreto, para los autores la interacción con herramientas digitales poco conocidas obstaculiza el proceso enseñanza-aprendizaje; advierten que, durante la fase inicial de intervención por medios audiovisuales, los participantes del grupo experimental se mostraron ansiosos por trabajar con equipos de cómputo que no conocían anteriormente (27).

Por otro lado, los resultados de nuestro trabajo difieren de las conclusiones reportadas en un estudio prepost intervención cuya estrategia educativa se basó en el enfoque colaborativo, a través del rompecabezas de Aronson; en esa investigación, la puntuación media de los cuestionarios de conocimiento fue de 3.8 en el pretest a 4.9 post-intervención, una diferencia significativa entre los dos momentos de la aplicación (11). Esta intervención contempló dos horas teóricas sobre lavado de manos, la revisión de una guía de práctica clínica y una sesión de práctica de dos horas supervisada por personal capacitado, incluyendo la técnica colaborativa del rompecabezas, para intercambiar conocimientos relacionados con diferentes modalidades de la higiene de manos (28).

También se advierte otra divergencia en la evaluación de una intervención educativa implementada con personal sanitario de un hospital en Valencia, España. Se trató de un taller presencial con sesiones semanales de una hora de duración sobre la higiene de manos, el uso racional de guantes y soluciones hidroalcohólicas; al respecto los autores determinaron el impacto positivo del taller en cuanto a la adquisición de conocimientos con más de un $70 \%$ de respuestas correctas con respecto al cuestionario aplicado para evaluar en escenarios prácticos, la disposición y el tipo de lavado correspondiente; sin embargo, esta evaluación se realizó posterior a los contenidos del taller sin disponer de un basamento que facilite la comparación $(29,30)$.
Se observa que los dos estudios citados previamente implementaron intervenciones educativas presenciales, a diferencia de nuestro trabajo. Es por eso, que el proceso de adaptación a nuevas modalidades educativas y el uso de herramientas tecnológicas puede representar un reto que compromete el desarrollo de una capacitación sobre el lavado de las manos. No obstante, concordamos con las observaciones que hacen referencia a la importancia de evaluar las intervenciones educativas sobre lavado de manos y se considera relevante que la evaluación implique una mirada retrospectiva para replantear estrategias pertinentes que provoquen el desarrollo cognitivo, contemplando la participación del estudiante y la intención de atender sus necesidades e intereses formativos (31).

\section{Conclusiones}

- La construcción del conocimiento en torno al cuidado del lavado de manos ha pasado por un proceso histórico y educativo que es paralelo con las corrientes pedagógicas predominantes en el aprendizaje de los cuidados de la enfermería. Con esta investigación, se pretende reflexionar sobre uno de los aspectos que a menudo son olvidados durante las intervenciones educativas: la evaluación del proceso.

- Conviene subrayar que en las investigaciones con enfoque constructivista los estudiantes tienen la oportunidad de aumentar el interés sobre los temas tratados, traspasar las barreras sobre la disponibilidad de la información, mejorar las actitudes $\mathrm{y}$ percepciones que tienen en torno a un tema y remarcar la importancia e impacto que tiene específicamente el lavado de manos, en la calidad de los cuidados y la vida de los pacientes. A pesar de que el objetivo de esta investigación recae específicamente en la efectividad respecto al aumento de conocimiento, se reconoce como una limitación la evaluación del proceso de enseñanza-aprendizaje con los estudiantes en comparación con otras metodologías, por lo que se considera una recomendación para futuras investigaciones.

- Desde otro punto de vista, se precisa que, aunque no hubo un aumento significativo del conocimiento en los estudiantes, existen varios factores que podrían explicar estos resultados, tales como: el medio virtual, la familiarización que los estudiantes tienen con el procedimiento y principalmente, porque su nivel de conocimiento ya se encontraba en un nivel alto. Se recomienda para futuras investigaciones utilizar métodos de enseñanza sobre esta temática relacionados con la enseñanza in situ, tales como 
Revista

CienciäoCuidado

Scientific Journal of Nursing

la capacitación dentro de los servicios de salud o la simulación

Conflicto de Intereses
Marco Esteban Morales-Rojas, Sheila Mariela Cohuo-Cob, Didier Francisco Aké-Canul, Russel Izael Trujeque-Zavala, Marianely

Pech-Irola

\section{Referencias Bibliográficas}

1. González M, Crespo S. Salud Pública y enfermería comunitaria. Primera ed. Morales J, editor. Ciudad de México: Manial Moderno; 2018.

2. Organización Mundial de la Salud. OMS. Una atención limpia es una atención segura. [Online].; 2019 [cited 2020 diciembre 14. Available from: https://www.who.int/gpsc/5may/es/

3. Organización Mundial de la Salud. Personal de Enfermería y de Partería, ¡Una atención limpia está en sus manos! [Online].; 2020 [cited 2020 diciembre 2. Available from: https:/www.who.int/infection-prevention/ campaigns/clean-hands/5may2020/es/

4. Astoray E, Condor D, Mendoza R. Nivel de conocimiento sobre el lavado de manos del personal de enfermería del Servicio de Pediatría de la Clínica Ricardo Palma, 2017. Tesis de Especialidad. Lima: Universidad Peruana Unión, Unidad de Posgrado de Ciencias de la Salud; 2017.

5. Martos M, Mota E, Martos R. Hand Hygiene Teaching Strategies among Nursing Staff: A Systematic Review. Int J Environ Res Public Health. 2019 Agosto; 16(17): p. 1-13.

6. Alzyood Mea. COVID-19 reinforces the importance of handwashing. Clinical Nursing. 2020 Agosto; 29(15-16): p. 2760-2761.

7. Organización Panamericana de la Salud. La higiene de manos en el momento adecuado salva vidas y es un indicador de la calidad y bioseguridad de los servicios de salud [OPS México]. México; 2017 [cited 2021 Enero. Available from: https://www.paho.org/mex/index.php?option=com content\&view=article\&id=1261:la-higiene-de-manos-en-el-momento-adecuado-salva-vidas-y-es-un-indicador-de-la-calidad-y-bioseguridad-de-los-servicios-de-salud\&Itemid=499

8. Xiong P, Zhang J, Wang X. Effects of a mixed media education intervention program on increasing knowledge, attitude, and compliance with standard precautions among nursing students: A randomized controlled trial. Am J Infect Control. 2017 Abril; 45(4): p. 389-395.

9. Dickie R, Rasmussen S, Cain R. The effects of perceived social norms on handwashing behaviour in students. Psychology, Health \& Medicine. 2017 Junio; 23(7): p. 1-6.

10. Organización Mundial de la Salud. Una atención más limpia es una atención más segura [OMS]. [cited 2021 Enero. Available from: https://www.who.int/gpsc/background/es/

11. Dembillo T, González V, Cervera A. Cooperative Learning and Hand Disinfection in Nursing Students. Nursing Research and Educatión. 2018 Junio; 36(2): p. 101-112.

12. Roman JAM. La educación superior en tiempos de pandemia: una visión desde dentro del proceso formativo. Revista Latinoamericana de Estudios Educativos. 2020 Septiembre; 50(ESPECIAL): p. 13-0.

13. Clinic Barcelona Hospital Universitario. ¿Qué es el Coronavirus SARS-CoV-2? [Portal Clinic].; 2020 [cited 2021 Enero. Available from: https://www.clinicbarcelona.org/asistencia/enfermedades/covid-19/definicion

14. Instituto Nacional de Estadística y Geografía. Estadísticas a próposito del Día Mundial del Internet (17 de mayo del 2020).; 2020 [cited 2021 Enero. Available from: https://www.inegi.org.mx/contenidos/saladeprensa/ aproposito/2020/eap internet20.pdf

15. Dirección General de Calidad y Educación en Salud. Herramientas y recursos de seguridad del paciente. [Online].; 2017 [cited 2020 marzo 18. Available from: http://www.calidad.salud.gob.mx/site/calidad/herramien- 
Revista

Cienciäocuidado

Scientific Journal of Nursing
Estrategia de Intervención digital basada en el constructivismo

para el conocimiento de lavado de manos en estudiantes de

enfermería

tas seguridad paciente.html

16. González J, Fernández M, Trujllo H. Cuestionario para evaluar en médicos conductas, conocimientos y actitudes sobre la higiene de manos. Gaceta Sanitaria. 2012 febrero; 26(5): p. 429-435.

17. Garzon L, Bautista V, Torres E. Prácticas y estrategias didácticas en el modelo pedagógico constructivista en enfermería. Revista Repertorio De Medicina Y Cirugía, 2020 Junio; 20(10), 30-35.

18. Mamani K. Relación entre conocimiento y práctica de higiene de manos en internos de enfermeria del hospital regional manuel Nuñez Butrón. Tesis de Grado. Puno, Perú: Universidad Nacional del Altiplano, Facultad de Enfermería; 2018.

19. Moran J, Gimeno A, Martínez E. Conocimiento de la higiene de manos en estudiantes de enfermería. Enfermería Global. 2014 Julio; 13(35).

20. Korhonen A, Vuori A, Lukkari A. Increasing nursing students' knowledge of evidence-based hand-hygiene: A quasi-experimental study. Nurse education in practice. 2019 Febrero; 35(1): p. 104-110.

21. Lira R. Conocimientos, Actitudes y Prácticas sobre el Lavado de Manos en el personal de salud del departamento de pediatría del Hospital escuela Oscar Danilo Rosales Arguello. 2019. Tesis de Especialidad. León: Universidad Nacional Autónoma de Nicaragua, Facultad de Ciencias Médicas; 2020.

22. González J, Fernández M, Trujillo H. Cuestionario para evaluar en médicos conductas, conocimientos y actitudes sobre la higiene de manos. Gaceta Sanitaria. 2012 septiembre-octubre; 26(5): p. 429-435.

23. Ricardo G, Castrejón V, González M. Conocimiento de los estudiantes de enfermería sobre las medidas de prevención en infecciones nosocomiales. Lux Médica. 2017 mayo-agosto; 12(36).

24. García Carpintero B, Siles González J, Martínez Roche M, Martínez Miguel E, Manso Perea C, Álvarez Embarba B. Metodologías de enseñanza-aprendizaje en enfermería: ¿Es el portafolio una metodología acorde con el Espacio Europeo de Educación Superior? Publicación semestral Enfermería Docente. 2017 Junio;(108).

25. Lima M, Carrión T, Herráiz A. Enfermería Comunitaria y salud digital en tiempos de la COVID-19. Ridec. 2020 Mayo; 13(2): p. 34-42.

26. Bloomfield J, Roberts J, Alison W. The effect of computer-assisted learning versus conventional teaching methods on the acquisition and retention of handwashing theory and skills in pre-qualification nursing students: a randomised controlled trial. International Journal of Nursing Studies. 2010 March; 47(3).

27. Konicki T, Miller E. Use of a simulation to examine differences in nursing students' hand hygiene knowledge, beliefs and behaviors. Nurse Education Today. 2016 June;(45).

28. Interial-Guzmán MG, Moreno-Monsiváis MG, Vázquez-Arreola L. Percepción y conocimiento de higiene de manos en personal de Enfermería. Publicación anual Políticas sociales sectoriales. 2020 Agosto 2020 - Julio 2021; 7(7).

29. Tenías JM, Mayordomo C, Benavent ML, Micó M, García-Esparza MdlÁ, et al,. Impacto de una intervención educativa para promover el lavado de manos y el uso racional de guantes en un hospital comarcal. Revista Calidad Asistencial. 2009 Octubre; I(24).

30. Amaya C. Conocimiento y la aplicación del lavado de manos en el personal asistencial del servicio de emergencia del Hospital la Merced - Chanchamayo - 2014. Tesis de Especialidad. Huánco, Perú: Universidad Nacional Hermilio Valdizán, Departamento de Enfermería; 2016.

31. Bloch-Melgarejo Y, Auña-Ramírez G, Oliveira H, Orué-Arce P. Cumplimiento del Protocolo de Lavado de Manos por profesionales de enfermería en un servicio de salud de la ciudad de Encarnación, marzo-julio del 2019. Publicación cuatrimestral Memorias del instituto de investigaciones en ciencias de la salud. 2020; 2(18). 